DOI: $10.2478 / v 10282-012-0013-2$

\title{
Neuroendoscopic surgery in hydrocephalus
}

\author{
H. Stan, P.A. Kiss, A. Stan, I.St. Florian \\ Neurosurgical Clinic, Clinical Emergency Hospital, Cluj-Napoca, Romania \\ Neurological Department, "CF” Hospital Cluj Napoca
}

\begin{abstract}
Hydrocephalus represents one of the most frequent pathologic entities requiring neurosurgical intervention. Even in present times the treatment of hydrocephalus is a highly debated subject. Neuroendoscopy is one of the technique posibility. The present study includes patients with different types of hydrocephalus classified according to imagistic criteria (MRI). Neuroendoscopic therapeutic options are presented for different types of hydrocephalus and the postoperative results of these procedures are revealed by imaging. In conclusion, we consider neuroendoscopy useful in each type of hydrocephalus.
\end{abstract}

Key words: hydrocephalus, neuroendoscopy, therapy

\section{Introduction}

Hydrocephalus is an active distension of the ventricular system in the brain resulting from inadequate passage of CSF from its point of production to its point of absorption into the systemic circulation. $(4,5)$

Currently the most common therapeutic options used to treat hydrocephalus are peritoneal drainage and neuroendoscopic surgery. Although there are pros and cons for each option, ventriculoperitoneal drainage techniques have a high complication rate. These complications are not rare and can be presented as range of signs and symptoms, which are well known. Highest frequency represented by drainage system obstruction and infection, despite the technical progress achieved in the drainage systems development. (2) Each of these complications require surgery to treat increasing the morbidity and mortality of this nosological entity. Neuroendoscopy is a valuable alternative in the treatment of hydrocephalus. $(1,6,7,9,10)$ If a drainage is done through a foreign body (drain, drain valve) to a place different from the natural physiological CSF absorption system (peritoneum, pleura, pericardium), the neuroendoscopic technique creates a "natural" shunting in the same physiological space, through the brain parenchyma. There are multiple neuroendoscopic procedures, adapted to each type of hydrocephalus, thus the endoscope can be used to decrease the production of cerebrospinal fluid by choroid plexus coagulation, to create other ways of CSF leakage (VCS) or to restore normal pathways of CSF leakage (apeductoplastia, septostomia, plasty of Magendi and Monroe foramens). (15) There are complications in neuroendoscopice techniques. Failure of endoscopic drainage can be represented by a poor CSF absorption or new obstructions in CSF drainage. Formation of a new arachnoid membrane plays a more important role in cases of endoscopic procedure failure, rather than poor CSF 
absorption. If an obstruction is not discovered, a ventriculoperitoneal drainage was done with the ventricular catheter placed in prepons tank under endoscopic guidance. (13) The main rationale is the fact that the ventricular catheter passes through multiple fixed cerebrospinal fluid circulation points in order to allow the free circulation of the cerebrospinal liquid.

\section{Material and methods}

In the period 2006-2012 in our service we performed a number of 110 endoscopic third ventriculostomys (ETV) to patients with different ages, in this sample being included 21 of ETV in children under 1 year (figure 1).

The average age of patients in the sample was 31.9 years (figure 2).

Children under one year of age ranged between 1 month and 12 months, the average being 5.3 months (figure 3 ).

There were 11 cases of ETV failure in patients over 1 year, in this cases we repeated the ETV or we did shunt conversion (figure 4).

A quarter of patients under one year resorted to ventriculoperitoneal drainage (figure 5).

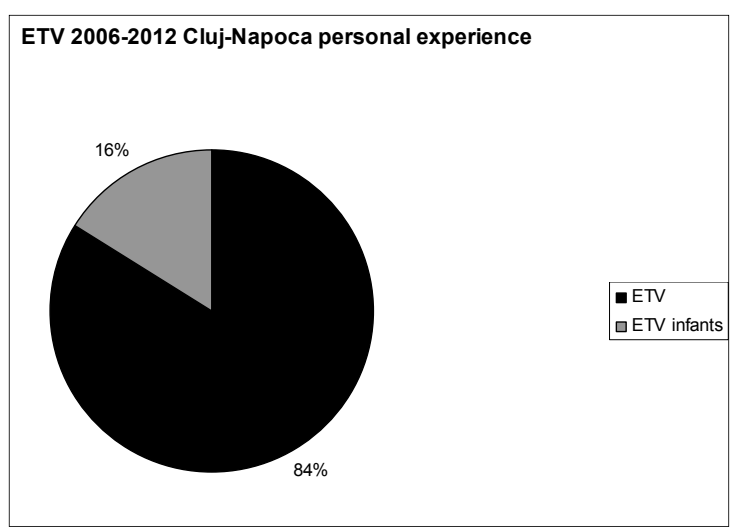

Figure 1 The percentage representation of ETV in children under 1 year

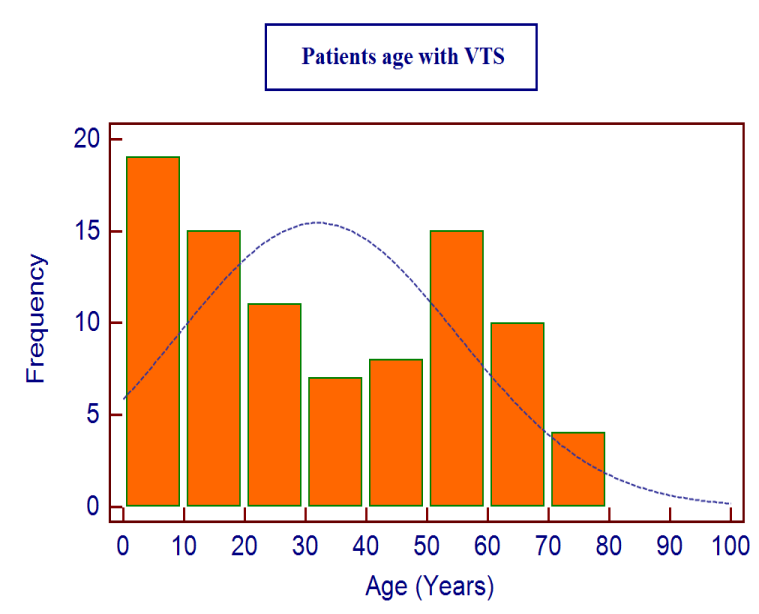

Figure 2 The histogram on the age groups of patients with VTE in the period 2006-2012

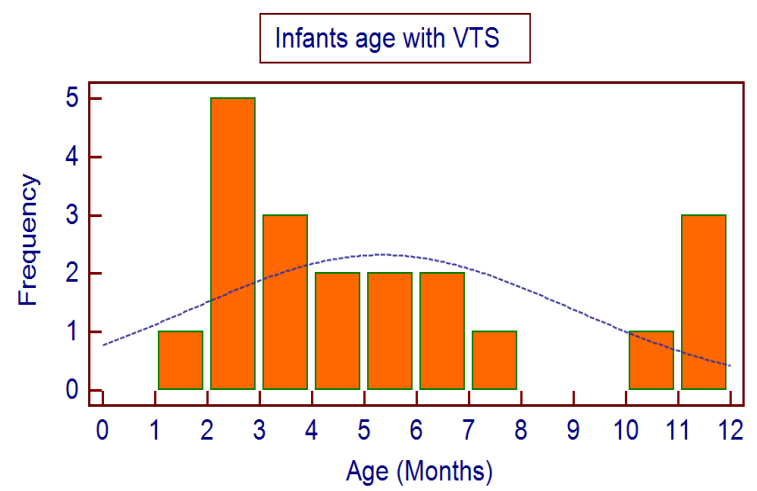

Figure 3 The histogram by age in children under one year with ETV during the 2006-2012

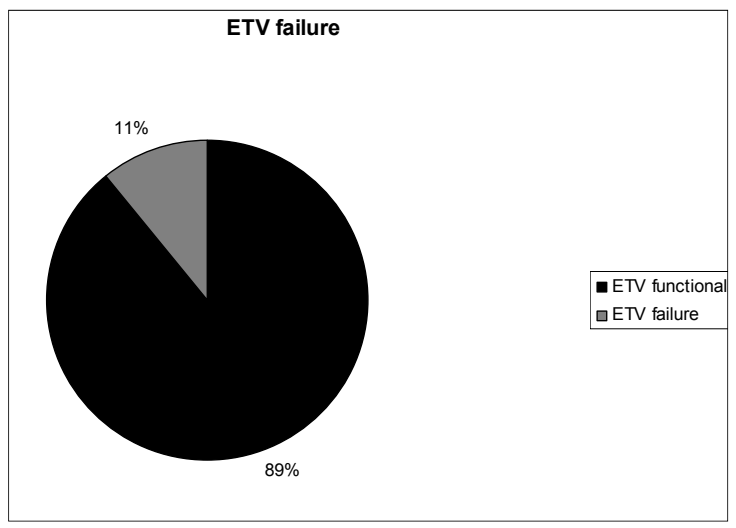

Figure 4 The representation of the percentage of patients aged over 1 year which initial ETV failed 


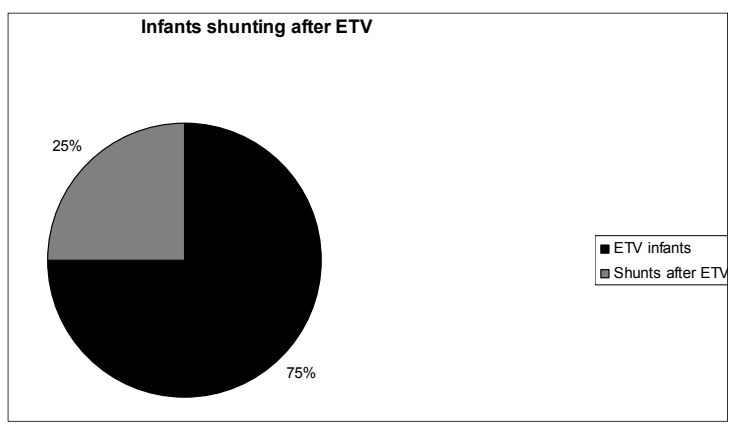

Figure 5 Percentage representation of patients under the age of one year which initial ETV failed

We used an Karl Storz endoskope with DECQ neuroendoscopy set.

All patients were investigatet using MRI or CT-scan.

MRI aspects have permitted the classification of hydrocephalus in several categories:

- Univentricular hydrocephalus

- Biventricular hydrocephalus

- $\mathrm{Bi}$ /triventricular hydrocephalus

- Triventricular hydrocephalus

- Tetraventricular hydrocephalus

- Communicating

- Occlusive

- Multilocular hydrocephalus

\section{Results and discussions}

\section{Occlusive hydrocephalus}

Univentricular:

- ventricular horn excluded

The cause is frequently represented by an intraventricular membrane that doesn't permit the CSF drainage towards the rest of the ventricle but other causes may also exist (inflammatory, neoplasm). (8) A large opening of the ventricular horn towards the ventricle can be made endoscopicaly (figure 7). The membrane can be excised using scissors and forcepses. An intraventricular cyst can also be the cause, originating in the choroid plexus or in the parietal ependima.

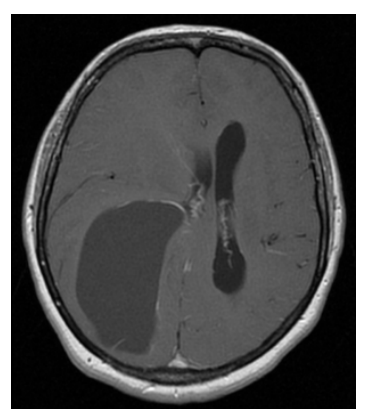

A

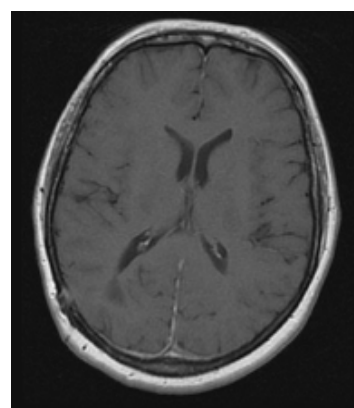

B

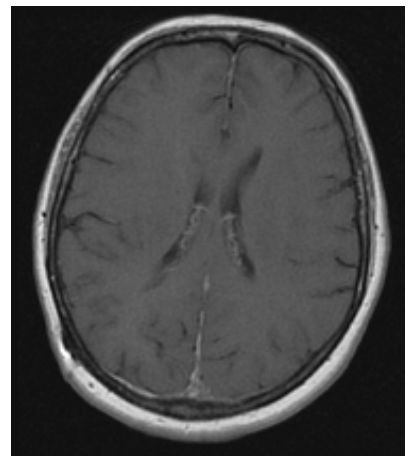

C

Figure 7 A Colloid cyst of the right lateral ventricle. Endoscopic excision of the cyst and its opening into the ventricular system is performed. $\mathbf{B}$ and $\mathbf{C}$ : postoperative control reveals the disappearance of the cyst and normal ventricular system. The burr hole of the approach is visible.

- Fourth ventricle excluded:

The purpose of surgery is placing a catheter in the Sylvius aqueduct. A $4 \mathrm{~cm}$ precoronal, mediopupillary line approach can be used only in those cases where the supratentorial ventricular system is also dilated. Another option is the cerebellous approach with Sylvius's aqueduct cannulation from the fourth ventricle.

\section{Biventricular}

In foramen of Monro stenosis recalibration with the Fogarty balloon (usually the right) and a septostomy is used (figure 8). 


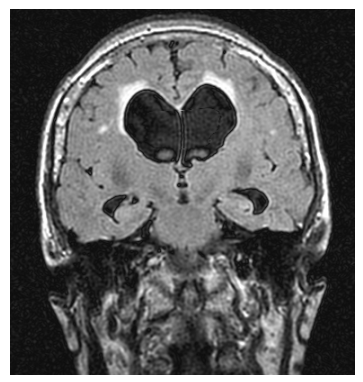

A

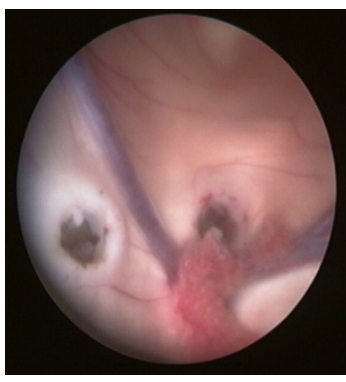

B

Figure $8 \mathrm{MRI}$ examination highlights the dilation of the lateral ventricles with signs of activity. $\mathbf{B}$ : septostomy and recalibrated foramen of Monro

\section{Bi-triventricular}

The lateral ventricles are dilated and to some degree, a dilatation of the third ventricle also exists. Stenosis of the Sylvian aqueduct and curving of the third ventricle floor with inferior convexity are evident. Endoscopically we can visualise the cause of the Monro orifice stenosis, recalibrate it and perforate the floor of the third ventricle (figure 9).

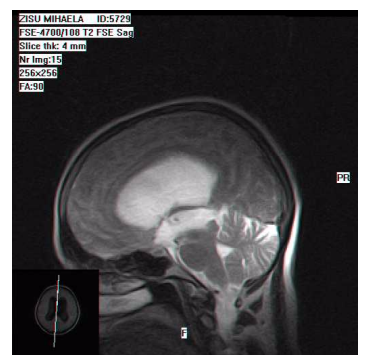

A

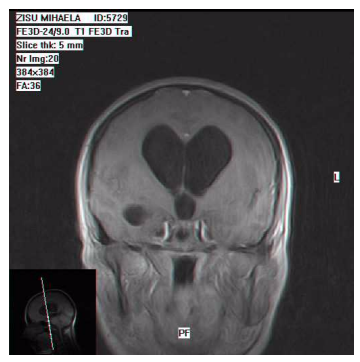

B

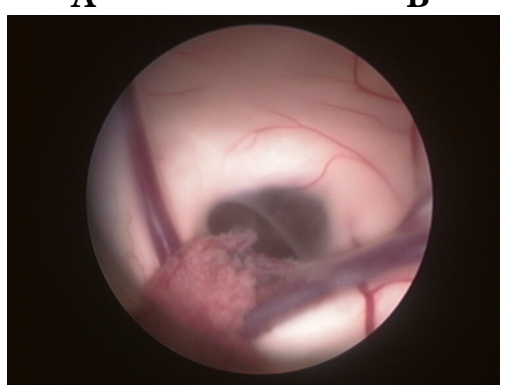

C

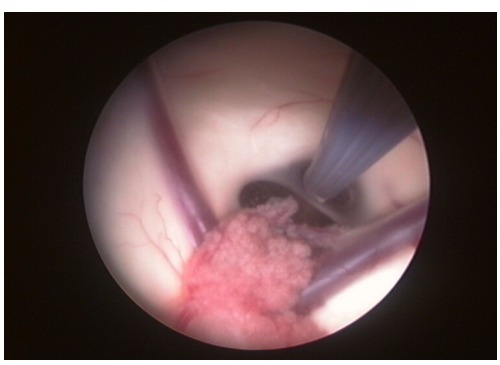

D

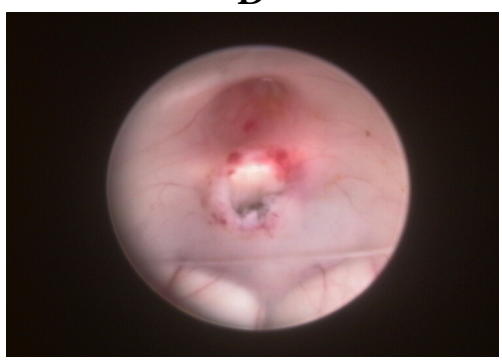

E

Figure 9 A, B MRI examination highlights the dilatation of the lateral ventricles and of the third ventricle with inferior bulging of its floor. C: Foramen of Monro with the lateral half occluded by an arachnoid membrane. D: Recalibration of the orifice of Monro. E: The perforated floor of the third ventricle

\section{Triventricular}

The stenosis of the aqueduct leads to the dilatation of the lateral ventricles and of the third ventricle.The floor of the third ventricle bulges thwards the inferior proving the existent pressure gradient between the third ventricle and the interpeduncular cistern. Classic ventriculocisternostomy is used (figure 10).

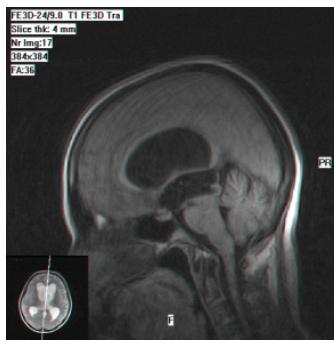

A

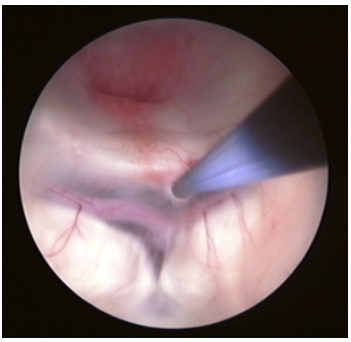

B 


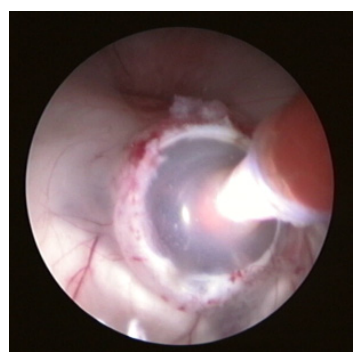

$\mathrm{C}$

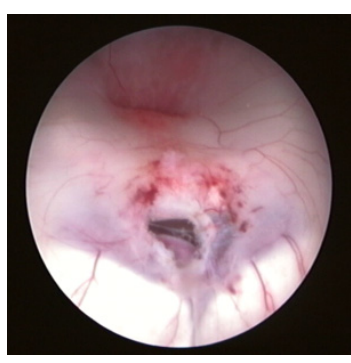

D
Figure 10 A MRI examination which reveals triventricular hydrocephalus caused by the stenosis of the aqueduct. B: perforation of the floor of the third ventricle $\mathbf{C}$ : dilatation of the orifice using the Fogarty balloon catheter $d$. final aspect

In the case of a child under one year old, the surgical technique has a few particularities. The incision is made at the level of the anterior fontanelle, with the same surgical landmarks as for an adult. The epicranial aponeurosis and the dura mater are prepared in such a way that they can be sutured at the end of the surgical intervention in order to reduce as much as possible the risk of a cerebrospinal fluid fistula. Ventriculocisternostomy is associated with coagulation of the choroid plexuses which can lead to decreased CSF production. In the absence of plexus coagulationg the success rate of the operation is significantly reduced. The coagulation can be performed in optimal conditions with the help of a flexible endoscope.

\section{Tetraventricular}

The obstacle in the way of CSF flow can be at the level of the foramen of Magendie or in other areas anterior to the interpedunculary cistern (figure 11).

In this case ventriculocisternostomy resolves the problem of hydrocephalus. The control MRI shows flow at the level of the stoma and decreasing of ventricular system size. The illustrated situation is yet another argument in favor of the necessity of evaluating patients with this pathology using the MRI.

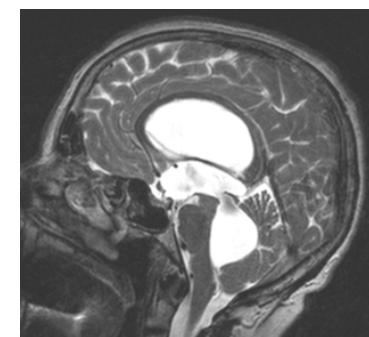

A

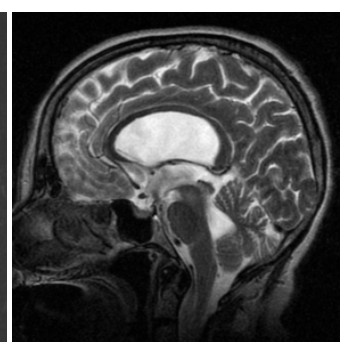

B
Figure 11 A Tetraventricular hydrocephalus caused by the occlusion of the foramen of Magendie.

Deformation of the third ventricular floor can be observed B: control-MRI 3 months postoperatively

\section{Communicating hydrocephalus}

Posthemorrhagic or postinfectious cases can be treated using endoscopic ventriculocisternosomy and coagulation of the choroid plexuses. In this case, a flexible endoscope is preferred. This permits the coagulation of the choroid plexus from the homolateral lateral ventricle, including the temporal horn, through the same burr hole which is used for the ventriculocisternostomy. After the septostomy the choroid plexus from the contralateral ventricle can also be entirely coagulated.

If we choose a ventriculoperitoneal shunting, the ventricular catheter can be placed under endoscopic control. After the ventriculocisternostomy the ventricular catheter is introduced so that its distal extremity passes through the stoma in the floor of the third ventricle. The interpeducular cistern, the third ventricle and the lateral ventricle will be drained through the lateral orifices of the catheter (figure 12). The catheter will remain in cerebrospinal fluid space regardless of the size of the ventricles, thus increasing its reliability. 

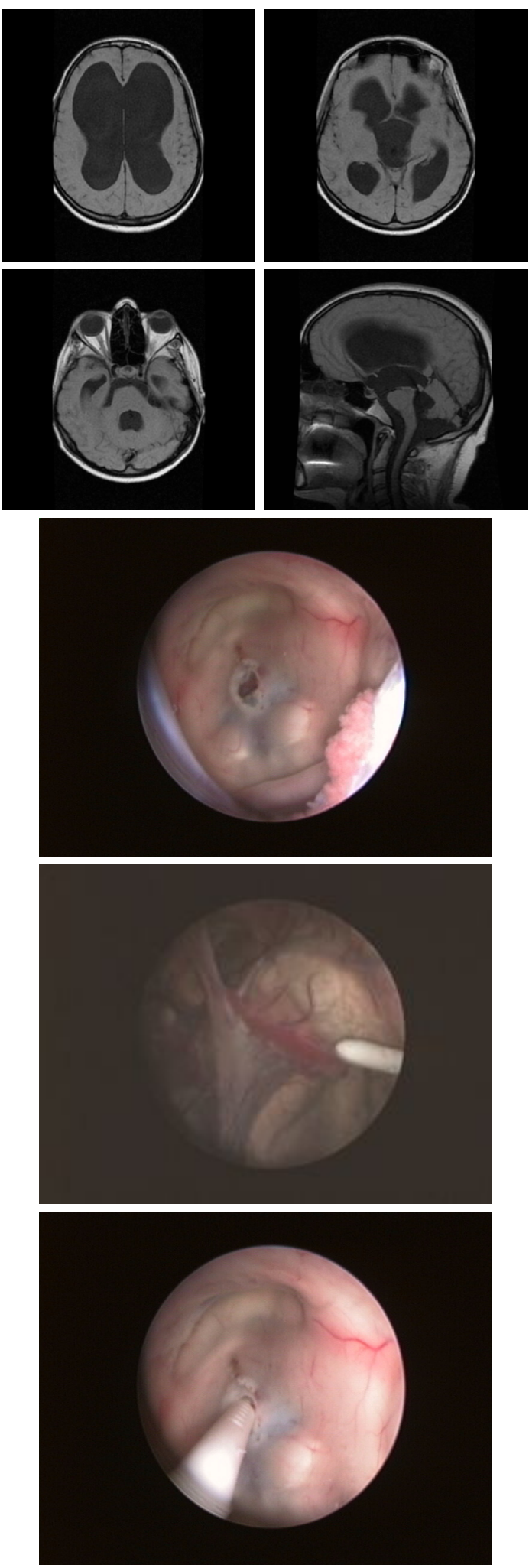

Figure 12 MRI revealed tetraventricular hydrocephalus. Ventriculocisternostomy followed by the insertion of the ventricular catheter under endoscopic control

\section{Multilocular}

It is usually postinfectious and it benefits from endoscopic approach because this technique allows the possibility of creating a communication between all of the existing compartments. Sometimes associated ventriculocisternostomy is enough but in some cases placing a ventricular catheter under endoscopic control for a ventriculoperitoneal shunting is also necessary. The catheter can be placed so that it drains one or more liquidian space.

\section{Particular situations}

When symptomatology does not improve after ventriculocisternostomy, endoscopic reintervention can be performed to assure the permeability of the orifice. There are two alternatives:

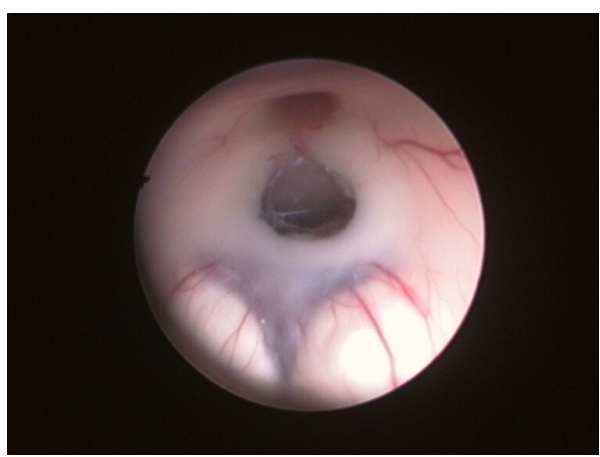

A

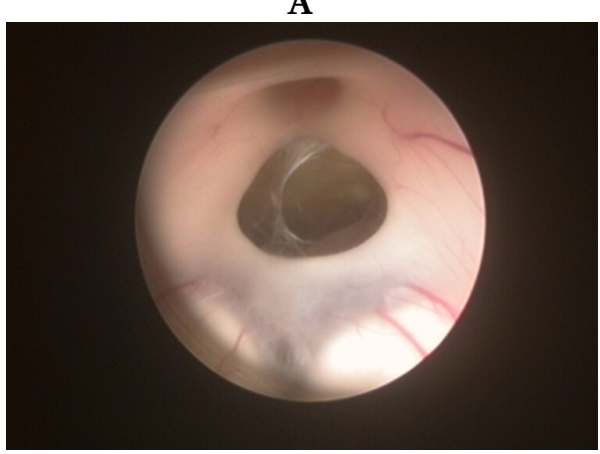

B 


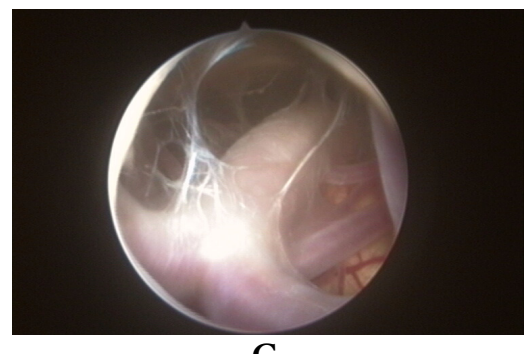

C

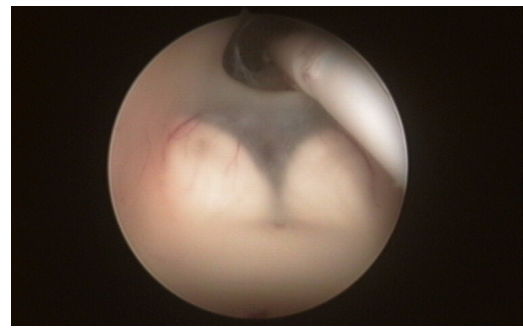

D

Figure 13 A final aspect after

ventriculocisternostomy $\mathbf{B}$ aspect of the stoma after 1 month $\mathbf{C}$ other arachnoid membranes under the floor are not highlighted d. placing of the ventricular catheter
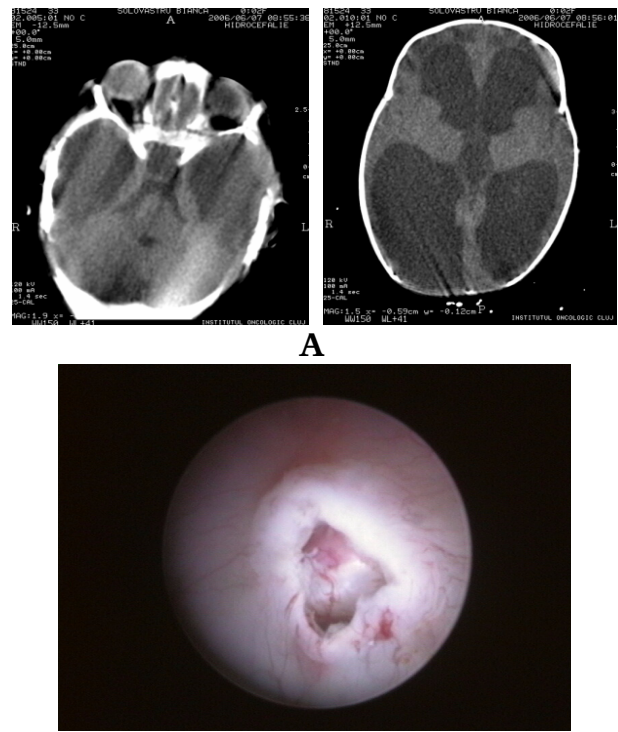

B

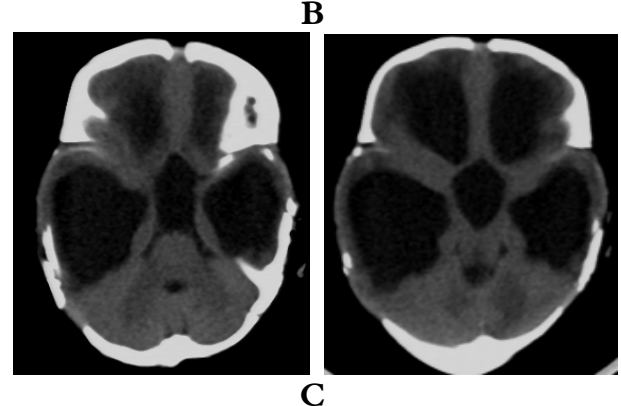

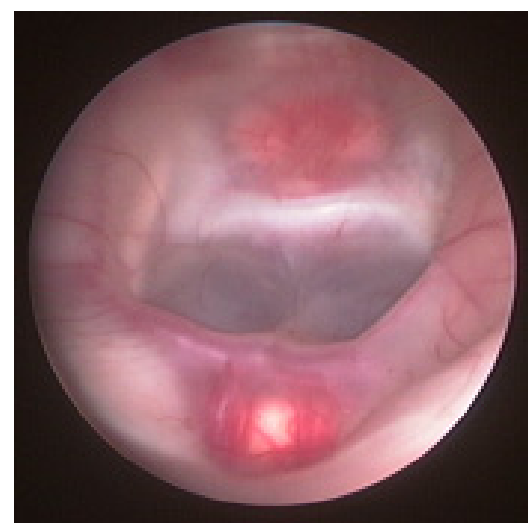

D

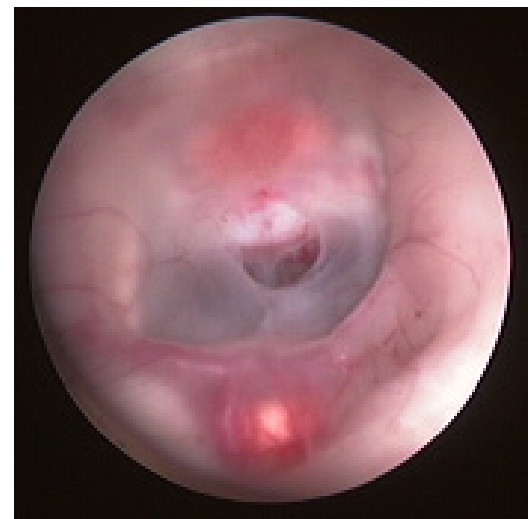

E

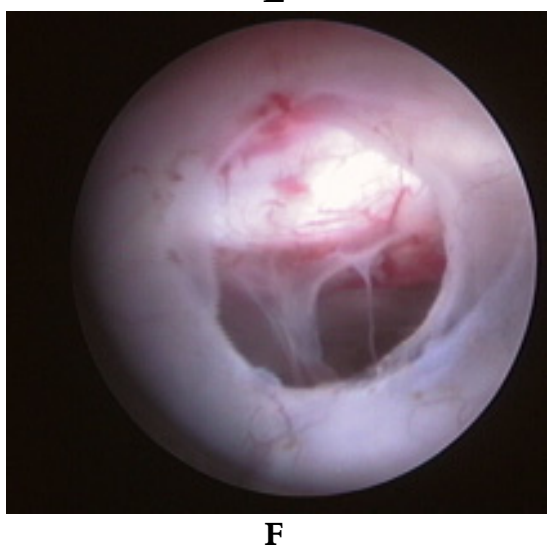

Figure 14 A Triventricular hydrocephalus - CT B aspect of stoma

C CT after 2 months d. endoscopic image of the floor e, f. lysis of the membranes with rebuilding of cerebrospinal fluid flow

If the orifice is occluded (most commonly by an arachnoid membrane), its fenestration and the rebuilding of CSF flow at the level of the stoma is performed 
(figure 14). The success rate of this operation reaches $90 \%$.

Follow-up of patients was made for one year. In our patients long-term shunt independence was achieved in $89 \%$ of patients, in independent literature longterm shunt independence vary much: $70 \%$, $77 \%$. $(11,3)$ The difference between the results can be made on behalf of the surgery indication in patients over 1 year where the diagnosis of hydrocephalus was based on clinical, neuroimaging and complementary tests (tap test). It is known that the therapeutic response efficacy varies depending on the selection of cases.(10) In the case of children under 1 year, long-term shunt independence was $75 \%$. The success of these patients being influenced much of the technique used. In the absence of plexus coagulationg the success rate of the operation is significantly reduced. The Warf BC study proving the difference between the cases where he practiced only ETV the succes rate was $48,6 \%$ compared to patients who practiced ETV and plexus coagulationg, where success has been $81,9 \%$. (14)

We believe that neuroendoscopic surgery is useful in any type of hydrocephalus.

\section{References}

1. Dirk F., Frank D., Hilmar K., Alexandra W. and Ernst G. Neuroendoscopic Tratament of Idiopathic Occlusion of the Foramen of Monro in Adults.Neurol Med Chir (Tokyo). 2002; 42:81-85.

2. Drake, James M. MB, BCh; Kestle, John R.W. MD; Milner, Ruth MSc; Cinalli, Giuseppe MD; Boop, Frederick MD; Piatt, Joseph Jr. MD; Haines, Stephen MD and Collaborators. Randomized Trial of Cerebrospinal Fluid Shunt Valve Design in Pediatric Hydrocephalus. Neurosurgery. 1998;43(2):294-303.

3. Dusick JR, McArthur DL, Bergsneider M. Success and complication rates of endoscopic third ventriculostomy for adult hydrocephalus: a series of 108 patients. Surg Neurol. 2008 Jan;69(1):5-15.

4. Harold L Rekate.The definition and classification of hydrocephalus: a personal recommendation to stimulate debate. Cerebrospinal Fluid Research. 2008; 5:2.

5. Hazel C Jones and Petra M Klinge.Hydrocephalus 2008, 17-20th September, Hannover Germany: a conference report. Cerebrospinal Fluid Research. 2008; 5:19.

6. Hideki Ogiwara \& Arthur J. Dipatri Jr. \& Tord D. Alden \& Robin M. Bowman \& Tadanori Tomita. Endoscopic third ventriculostomy for obstructive hydrocephalus in children younger than 6 months of age. Childs Nerv Syst.2010; 26:343-347.

7. Joachim M. K. Oertel \& Yvonne Mondorf \& Henry W. S. Schroeder \&Michael R. Gaab. Endoscopic diagnosis and treatment of far distal obstructive hydrocephalus.Acta Neurochir. 2010;152:229-240.

8. Kumar R. Unilateral hydrocephalus in paediatric patients, a trial of endoscopic fenestration. Neurol India .1999;47:282.

9. Nasser M. F. El-Ghandour. Endoscopic third ventriculostomy versus ventriculoperitoneal shunt in the treatment of obstructive hydrocephalus due to posterior fossa tumors in children. Childs Nerv Syst. 2010;1:1-10.

10.Paidakakos N, Borgarello S, Naddeo M. Indications for endoscopic third ventriculostomy in normal pressure hydrocephalus. Acta Neurochir Suppl. 2012;113:123-7.

11.Rahme R, Rahme RJ, Hourani R, Moussa R, Nohra G, Okais N, Samaha E, Rizk T. Endoscopic third ventriculostomy: the Lebanese experience. Pediatr Neurosurg. 2009;45(5):361-7. Epub 2009 Nov 11.

12.Sandrine de Ribaupierre \& B. Rilliet \& O. Vernet \& L. Regli \& J.-G. Villemure. Third ventriculostomy vs ventriculoperitoneal shunt in pediatric obstructive hydrocephalus: results from a Swiss series and literature review. Childs Nerv Syst. 2007; 23:527-533.

13.Stan H, Popa C, Iosif A,Nistor S. Combined endoscopically guided third ventriculostomy with prepontine cistern placement of the ventricular catheter in a ventriculo-peritoneal shunt: technical note. Minim Invasive Neurosurg.2007;50:247-50.

14. Warf BC, Tracy S, Mugamba J. Long-term outcome for endoscopic third ventriculostomy alone or in combination with choroid plexus cauterization for congenital aqueductal stenosis in African infants. J Neurosurg Pediatr. 2012 Aug;10(2):108-11.

15. Yavor Enchev \& Shizuo Oi. Historical trends of neuroendoscopic surgical techniques in the treatment of hydrocephalus. Neurosurg Rev.2008; 31:249-262. 